\title{
Is Cystic Fibrosis Mucus Abnormal?
}

\author{
MALCOLM KING ${ }^{(11)}$ \\ Meakins Christie Laboratories, McGill University Clinic, Royal Victoria Hospital, Montreal, Quebec,
} Canada H3A $2 B 4$

\begin{abstract}
Summary
It has been known for some time that the viscosity of sputum from cystic fibrosis (CF) patients differs from that in other disease types characterized by pulmonary hypersecretion. However, it has never been established that there is in fact any abnormality in the rheologic properties of CF sputum. We have recently developed techniques to examine the rheology and mucociliary transportability of tracheal secretions from dogs utilizing the small quantities of mucus available from a healthy animal. In the present study, these in vitro techniques were applied to sputum samples obtained from 15 adult $C F$ patients. For mucoid and mucopurulent $C F$ sputum samples, the viscoelastic properties were remarkably similar to those observed for control canine tracheal mucus samples. For purulent CF sputum, elasticity and viscosity tended to be higher and the viscosity/elasticity ratio lower than for either the nonpurulent sputum or the canine tracheal mucus. The logarithm of elasticity at $1 \mathrm{rad} / \mathrm{sec}$ was $2.55 \pm 0.35$ (S.D.) for purulent sputum, $2.05 \pm 0.49$ for mucoid and mucopurulent sputum, and $2.17 \pm 0.36$ for canine tracheal mucus.

From the viscoelastic properties of CF sputum, one would have predicted the in vitro mucociliary transportability, at least for the nonpurulent samples, to be the same as that of canine tracheal mucus. However, the frog palate assay indicated a consistently lower rate of transport (mean difference, 13\%) than that predicted from previous studies. This result would, therefore, suggest that if the prediction from canine tracheal mucus is applicable there is a factor in the CF sputum that results in a temporary inhibition of frog palate ciliary beating. This apparent inhibition of mucociliary transport is, however, relatively minor, and it is perhaps more remarkable that for none of the sputum samples collected was the in vitro ciliary transport rate particularly low; i.e., observed values ranged from 65 to $100 \%$ of frog palate control.
\end{abstract}

\section{Speculation}

There is probably no rheologic abnormality in the tracheal mucus of patients with cystic fibrosis other than that associated with purulence. Cystic fibrosis sputum can be moved reasonably well by ciliary action, but there is, nevertheless, some suggestion of the presence of a ciliary inhibitory factor in it.

The question of the nature of the secretory abnormality in cystic fibrosis (CF) has troubled researchers for years. Sputum from $C F$ patients has been commonly described by such qualitative terms as "tenacious" or "thick." However, it has never been established that there is in fact any abnormality in the rheological properties of CF sputum.

It has been known for some time that the viscosity of sputum from CF patients differs from that in other disease types characterized by pulmonary hypersecretion. Sturgess, in 1969 (9), and Feather and Russell, in 1970 (2), reported that the mean viscosity of mucoid sputum from CF patients was less than that from patients with chronic bronchitis (CB), and, in the latter study, asthma. Both studies also reported that viscosity of purulent $C F$ sputum did not differ from that of purulent sputum from bron- chiectasis patients. Charman and Reid (1), in a broader study of sputum viscosity, substantiated these earlier findings. They showed that for mucoid CF sputum the viscosity was less than that of CB, bronchiectasis, or asthma sputum, whereas for purulent samples, no significant differences between disease types were seen. More rcently, Picot et al. (7) reported that for all macroscopic sputum types (mucoid, mucopurulent, and purulent) the CF sputum viscosity was significantly less than that of CB sputum. LopezVidriero and Reid (6), on the other hand, found only a trend toward lower viscosity for CF sputum with respect to CB sputum in the three macroscopic types. Although these studies differ in their fine details, they all agree that the mean sputum viscosity in $\mathrm{CF}$ is, if anything, lower than that in any of the other hypersecretory disease types, contrary to what had been anticipated.

None of the above work has established, however, that there is no rheologic abnormality in CF sputum. First of all, there was no control from healthy subjects because healthy subjects do not spontaneously produce sputum. Secondly, only viscosity, and not viscoelasticity, was measured, and, furthermore, in most of the work, the viscosity was determined at shear rates $\left(1350 \mathrm{sec}^{-1}\right)$ that are probably well beyond those operative in ciliary flow. It is possible that the rheologic abnormality would only appear in the elasticity or perhaps in the interrelation of viscosity and elasticity.

\section{MATERIALS AND METHODS}

We have recently developed a technique for the rheologic examination of tracheal secretions from dogs utilizing the small quantities of mucus available from a healthy animal (5). Mucus is generally collected from tracheostomized, conscious dogs because of the inhibitory effect of anesthesia on mucociliary flow (4). Briefly, the technique involves inserting a cytology brush through the tracheostomy (which is normally closed off to the atmosphere) and allowing it to rest in contact with the trachea for a period of 2 or $3 \mathrm{~min}$, during which time mucus flows on to the brush and saturates it. The mucus (generally a few microliters) is then removed from the brush and transferred to a sample container for subsequent rheologic examination. This is accomplished in a magnetic microrheometer (5), in which a small steel sphere (approximately $100 \mu$ ) inserted in the drop of mucus is vibrated by means of an electromagnet. The amplitude of vibration and the phase lag with respect to the driving force are used to calculate the elasticity and viscosity at each driving frequency.

The samples of canine tracheal mucus are also subjected to another in vitro assay-mucociliary transportability. This is done by placing the tracheal mucus samples on a mucus-depleted, cilioactive frog palate (an epithelium with active cilia but no mucus) and comparing its rate of transport with that of native frog palate mucus. This assay along with the viscoelastic properties led to a correlation between mucociliary transportability and viscoelasticity of canine tracheal mucus (3). Specifically, it was found that transportability decreased with both the elasticity and the viscosity (which were themselves strongly correlated) as well as with the viscosity/elasticity ratio (which decreased moderately with increasing elasticity). From this study, a predictive function 
relating transportability with viscoelasticity was established as follows:

$$
\operatorname{Tr}=1.612-0.2211 \log G^{\prime}(1)-0.7678 \tan \delta(1)
$$

where $\operatorname{Tr}$ is the normalized rate of mucus transport (relative to frog palate control), $\mathrm{G}^{\prime}(1)$ is the mucus elasticity (determined at a frequency of $1 \mathrm{rad} / \mathrm{sec})$, and $\tan \delta(1)$ is the viscosity/elasticity ratio of the mucus (at $1 \mathrm{rad} / \mathrm{sec})$.

- In the present study, the in vitro techniques described above were applied to sputum samples obtained from 15 adult CF patients attending an outpatient clinic. All samples were collected between 10 and $12 \mathrm{AM}$ and stored in a refrigerator until analyzed within 3 hours.

Each sample was characterized (1) according to its macroscopic appearance, i.e., mucoid (no evidence of pus), purulent (uniformly purulent, whether green or yellow), or mucopurulent (mixed). No attempt was made to homogenize the samples. Where sputa were visibly nonhomogeneous, aliquots were taken from both solidand liquid-like phases, although material that had the appearance of saliva was avoided. Two to four aliquots of 5 to $10 \mu \mathrm{l}$ were taken from each sputum sample. Each aliquot was analyzed in the same fashion as described above, i.e., its elasticity and viscosity were determined over a range of frequencies, and its mucociliary transportability was assayed on the frog palate.

\section{RESULTS}

The sputum samples were grouped into two classes: mucoid (including two mucopurulent samples) and purulent. The viscoelastic results are summarized in Figures 1 and 2. Figure 1 shows the elasticity $G^{\prime}$ and viscosity $\eta^{\prime}$, measured at 1 radian/sec, for individual CF sputum samples, and the previously reported mean data for canine tracheal mucus. Figure 2 shows $\tan \delta$ (= frequency $X$ viscosity/elasticity) at $1 \mathrm{rad} / \mathrm{sec}$ for the same samples. For the mucoid and mucopurulent CF sputum samples, the viscoelastic properties were remarkably similar (mean and range) to those observed for control canine tracheal mucus samples. This was the case for both $G^{\prime}$ and $\eta^{\prime}$, as shown in Figure 1, and for $\tan \delta$, as shown in Figure 2. Viscoelastic measurements made at 10 and 100 $\mathrm{rad} / \mathrm{sec}$ showed similar overlap between nonpurulent CF sputum and canine tracheal mucus.

For purulent CF spsutum, $G^{\prime}$ and $\eta^{\prime}$ tended to be higher and $\tan \delta$ tended to be lower than for either the nonpurulent sputum or the canine tracheal mucus. Only the difference in $G^{\prime}$ between purulent $\mathrm{CF}$ sputum and normal canine mucus reached statistical significance $(P<0.05$ by both standard $t$ test and median test),

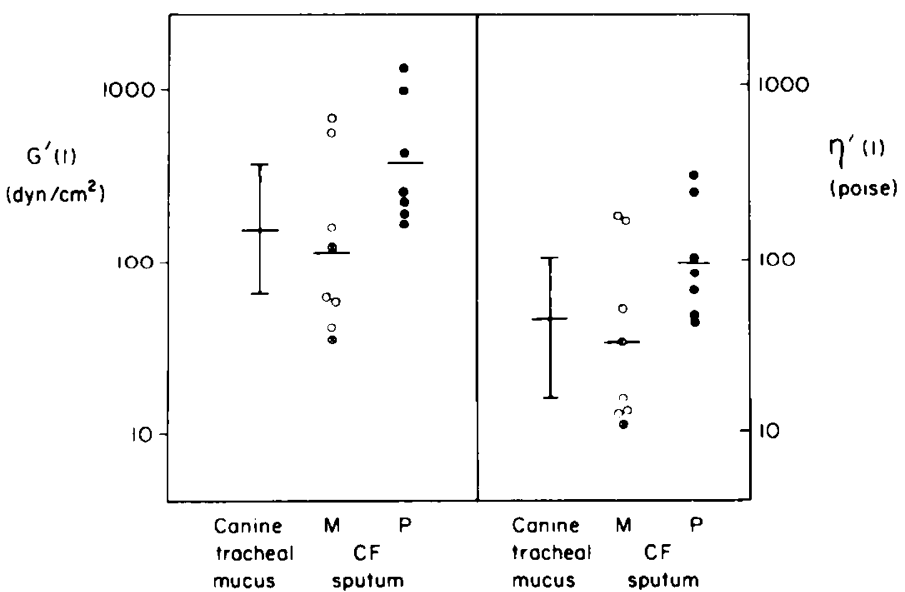

Fig. 1. Elasticity, $G^{\prime}$, and viscosity, $\eta^{\prime}$, measured at $1 \mathrm{rad} / \mathrm{sec}$, for CF sputum. $\bigcirc$, mucoid; $\otimes$, mucopurulent (grouped together as $\mathbf{M}$ ); $\odot$, purulent (plotted separately as $P$ ). Mean values \pm 1 S.D. for 102 control canine tracheal mucus samples also shown (from Ref. 3 ). principally because of the large number of canine samples included in the comparison. The trend to higher levels of elasticity and viscosity in the purulent sputum is consistent with previously published work (6). Perhaps more notable, however, is the degree of overlap between the data for purulent sputum and normal canine mucus, i.e., the elasticity and viscosity exceeded the means for canine mucus by more than 2 standard deviations only for one purulent sample.

From the viscoelastic properties of CF sputum, one would have predicted the in vitro mucociliary transportability, at least for the nonpurulent samples, to be the same as that of canine tracheal mucus. However, the frog palate assay indicated a consistently lower rate of transport than that predicted by equation (1). This is demonstrated in Figure 3, in which the observed frog palate transport rates are plotted as a function of the predicted rates. In every case, for both mucoid and purulent sputum the observed transport rate fell below the line of identity; the mean difference between observed and predicted (13\%) was highly significant. This result would, therefore, suggest that if the prediction from canine tracheal mucus is applicable there is a factor in the CF sputum that results in a temporary inhibition of frog palate ciliary beating. (It would have to be temporary because frog palate controls were taken before and after the aliquots of sputum were tested, and the control rate did not change. Furthermore, such an inhibitory factor would have to be rapidly diffusible to inhibit transport in this manner.)

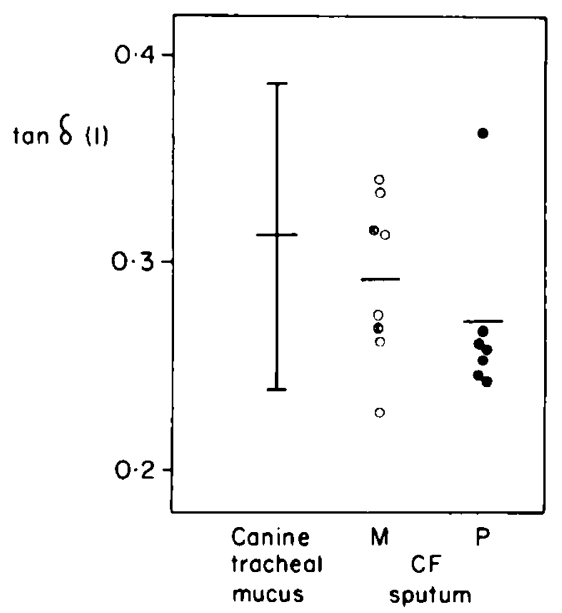

Fig. 2. Tan $\delta$ (viscosity/elasticity) measured at $1 \mathrm{rad} / \mathrm{sec}$ for CF sputum and canine tracheal mucus. Symbols as in Fig. 1.

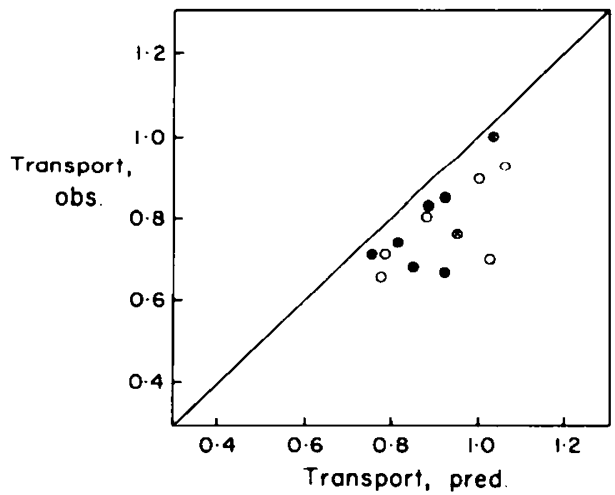

Fig. 3. Observed ciliary transport rate of CF sputum on frog palate, relative to native frog palate mucus, versus rate predicted from sputum viscoelasticity properties. $\bigcirc$, mucoid; $\otimes$, mucopurulent; $\bullet$, purulent. - line of identity. 


\section{DISCUSSION}

In summary, this study presents evidence to suggest that if there is a rheologic abnormality in CF sputum, it is limited to samples that are frankly purulent. It should be emphasized, however, that the present work does not constitute conclusive proof of this point. The procedures for the collection and handling of the CF sputum samples were not identical to the procedures used for the canine samples, and it may be invalid to compare human sputum with canine tracheal mucus. The final answer to this must await the collection of rheologically unaltered mucus from healthy humans.

The frog palate work suggests that there is a rapidly diffusible component of the CF sputum that inhibits the beating of frog palate cilia. It does not necessarily mean that there would be inhibition of human tracheal cilia, nor does it associate this factor with any particular chemical moiety. This apparent inhibition of mucociliary transport is in fact relatively minor, and it is perhaps more remarkable that for none of the sputum samples collected was the in vitro ciliary transport rate particularly low; i.e., observed values ranged from 65 to $100 \%$ of frog palate control. In the study by Puchelle $e t$ al. (8), in vitro ciliary transport rates for sputum from patients with chronic bronchitis were as low as $10 \%$ of control.

\section{REFERENCES AND NOTES}

1. Charman, J., and Reid, L.: Sputum viscosity in chronic bronchitis, bronchiectasis, asthma, and cystic fibrosis. Biorheology, 9: 185 (1972).

2. Feather, E. A., and Russell, G.: Sputum viscosity in cystic fibrosis of the pancreas and other pulmonary diseases. Br. J. Dis. Chest, 64: 192 (1970).

3. King. M.: Interrelation between mechanical properties of mucus and mucociliary transport: effect of pharmacologic interventions. Biorheology. 16: 57 (1979).

4. King, M., Engel, L. A., and Macklem. P. T.: Effect of pentobarbital anesthesia on rheology and transport of canine tracheal mucus. J. Appl. Physiol., 46: 504 (1979).

5. King, M., and Macklem, P. T.: Rheological properties of microliter quantities of normal mucus. J. Appl. Physiol., 42: 797 (1977).

6. Lopez-Vidriero, M. T., and Reid, L.: Chemical markers of mucous and serum glycoproteins and their relation to viscosity in mucoid and purulent sputum from various hypersecretory diseases. Am. Rev. Respir. Dis., 117: 465 (1978).

7. Picot, R., Das, I., and Reid. L.: Pus deoxyribonucleic acid, and sputum viscosity. Thorax, 33: 235 (1978).

8. Puchelle, E., Girard, F., and Zahm. J. M.: Rheology of bronchial secretions and mucociliary transport. Bull. Eur. Physiopathol. Respir. 12: 771 (1976).

9. Sturgess, J.: A new pattern of sputum viscosity. In: Proceedings of the 5th International Cystic Fibrobis Conference, Cambridge, p. 368 (1969).

10. The author is grateful to Naima Viires for her technical assistance and to Dr. Paul Despas for his collaboration in providing the sputum samples.

11. Requests for reprints should be addressed to: Dr. Malcolm King, Meakins Christie Laboratories, McGill University, 3775 University Street. Montreal, Quebec, Canada H3A 2B4.

12. Received for publication April 22, 1980

13. Accepted for publication July 16, 1980. 